\title{
Erratum: CTCF-mediated functional chromatin interactome in pluripotent cells
}

Lusy Handoko, Han Xu, Guoliang Li, Chew Yee Ngan, Elaine Chew, Marie Schnapp, Charlie Wah Heng Lee, Chaopeng Ye, Joanne Lim Hui Ping, Fabianus Mulawadi, Eleanor Wong, Jianpeng Sheng, Yubo Zhang, Thompson Poh, Chee Seng Chan, Galih Kunarso, Atif Shahab, Guillaume Bourque, Valere Cacheux-Rataboul, Wing-Kin Sung, Yijun Ruan \& Chia-Lin Wei Nat. Genet. 43, 630-638 (2011); published online 19 June; corrected after print 11 July 2011

In the version of this article initially published, the accession codes section contained inaccuracies. The raw sequences and processed data generated from this study can be downloaded with accession number GSE28247. The previously published histone modification data used in this study are found under accession numbers GSE12241 and GSE11172. The error has been corrected in the HTML and PDF versions of the article.

\section{Corrigendum: A cooperative microRNA-tumor suppressor gene network in acute T-cell lymphoblastic leukemia (T-ALL)}

Konstantinos J Mavrakis, Joni Van Der Meulen, Andrew L Wolfe, Xiaoping Liu, Evelien Mets, Tom Taghon, Aly A Khan, Manu Setti, Pieter Rondou, Peter Vandenberghe, Eric Delabesse, Yves Benoit, Nicholas B Socci, Christina S Leslie, Pieter Van Vlierberghe, Frank Speleman \& Hans-Guido Wendel

Nat. Genet. 43, 673-678 (2011); published online 5 June; corrected after print 11 July 2011

In the version of this article initially published, the name of author Manu Setty was incorrectly spelled as Manu Setti. The error has been corrected in the HTML and PDF versions of the article. 